\title{
Risk of multiple pancreatic cancers in CDKN2A-p16-Leiden mutation carriers
}

\author{
Isaura Ibrahim $\mathbb{1}^{1} \cdot$ Babs G. Sibinga Mulder ${ }^{2,3} \cdot$ Bert Bonsing $^{2} \cdot$ Hans Morreau $^{3}$ - Arantza Farina Sarasqueta ${ }^{3}$.

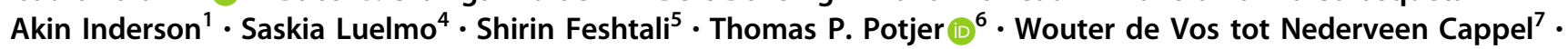 \\ Martin Wasser ${ }^{5} \cdot$ Hans F. A. Vasen ${ }^{1}$
}

Received: 22 December 2017 / Revised: 4 April 2018 / Accepted: 11 April 2018 / Published online: 16 May 2018

(c) European Society of Human Genetics 2018

\begin{abstract}
CDKN2A-p16-Leiden mutation carriers have a substantial risk of developing pancreatic ductal adenocarcinoma (PDAC). One of the main clinical features of hereditary cancer is the development of multiple cancers. Since 2000, we have run a surveillance program for CDKN2A-p16-Leiden mutation carriers. The patients are offered a yearly MRI with optionally endoscopic ultrasound. In patients with a confirmed lesion, usually, a partial resection of the pancreas is recommended. A total of 18 PDAC (8.3\%) were detected in 218 mutation carriers. In this report, we describe two CDKN2A-p16-Leiden patients with a synchronous and metachronous PDAC. Including two previously-reported cases, we identified four patients with multiple PDAC: two of 18 patients within the surveillance program (11\%) and two patients with a proven CDKN2A-p16-Leiden mutation not participating in the surveillance program. In conclusion, this study demonstrated a high risk of developing multiple PDAC in CDKN2A-p16-Leiden mutation carriers. After detecting a primary tumor, it is very important to exclude the presence of a second synchronous tumor. Moreover, after a partial pancreatectomy for PDAC, close surveillance is necessary. In view of the current findings, offering a total pancreatectomy might be an appropriate option in patients with an early PDAC.
\end{abstract}

\section{Introduction}

Hereditary factors account for 3-5\% of all pancreatic ductal adenocarcinomas (PDAC) [1]. In approximately $4 \%$ of

Isaura Ibrahim

i.s.ibrahim@lumc.nl

1 Department of Gastroenterology \& Hepatology, Leiden University Medical Center, Leiden, The Netherlands

2 Department of Surgery, Leiden University Medical Center, Leiden, The Netherlands

3 Department of Pathology, Leiden University Medical Center, Leiden, The Netherlands

4 Department of Oncology, Leiden University Medical Center, Leiden, The Netherlands

5 Department of Radiology, Leiden University Medical Center, Leiden, The Netherlands

6 Department of Clinical Genetics, Leiden University Medical Center, Leiden, The Netherlands

7 Department of Gastroenterology \& Hepatology, Isala Clinics, Zwolle, The Netherlands familial PDAC, a cancer is caused by an underlying defect in genes including BRCA2, CDKN2A, PALB2, the mismatch repair (MMR) genes, and STK11 [2]. The lifetime risk of developing PDAC for carriers of a gene defect in CDKN2A-p16-Leiden is 15-20\% [3].

One of the main features of hereditary cancer is the high risk of developing multiple (synchronous or metachronous) cancer. However, multiplicity might be masked in hereditary PDAC because most patients die within 6-12 months [4].

Recently, we described two patients with a $C D K N 2 A$ pl6-Leiden mutation with metachronous PDAC $[5,6]$. In the current report, we describe two additional $C D K N 2 A$ p16-Leiden patients, one with a synchronous and one with a metachronous PDAC.

\section{Patients and methods}

Since 2000, we have run a surveillance program in the Leiden University Medical Center (LUMC) for individuals with a founder mutation in the CDKN2A gene, called p16Leiden (NM_000077.4: c.225_243del19, p. 
(Ala76Cysfs*64)) [7]. The program consists of annual magnetic resonance imaging (MRI) and magnetic resonance cholangiopancreatography (MRCP), and, optionally, endoscopic ultrasound (EUS). In patients with a suspicious lesion, additional EUS and computed tomography (CT) scanning is performed within two to three weeks. If the lesion is confirmed by two out of the three modalities, surgery is offered. To avoid the serious morbidity associated with total pancreatectomy, until recently a partial resection of the pancreas was recommended. Acquired data was submitted to a public $C D K N 2 A$ gene variant database (https://databases.lovd.nl/shared/genes/CDKN2A; submission IDs \# 00155215 and \# 00155216).

\section{Results}

A total of 218 CDKN2A-p16-Leiden mutation carriers are under surveillance as of 01-04-2017. Since 2000, the screening program has detected PDAC in 18 patients $(8.3 \%)$ and nine of these patients have since died from metastatic disease. The mean follow-up time for all 18 patients is 24.1 months (median follow-up: 17.5 months).

The first patient with multiple PDAC was a 67-year-old female carrier of a CDKN2A-p16-Leiden mutation that first entered MRI/MRCP pancreas surveillance in 2006. The patient had a sister who died of PDAC at the age of 52. She reports drinking 1-2 glasses of wine a week and has never smoked. In 2016, 11 months after a previous normal MRI scan, the patient presented with painless jaundice. A new MRI scan showed a 14-mm lesion in the head of the pancreas, which was confirmed by EUS. The patient underwent a pancreaticoduodenectomy, and histopathological examination of the surgical specimen showed a moderately differentiated PDAC of $13 \mathrm{~mm}$, and one tumorpositive lymph node out of the 18 removed. After surgery, the patient was treated with gemcitabine and capecitabine. Regrettably, 6 months later, a yearly review of all MRI scans of patients in the surveillance program revealed that this patient had a second tumor in the corpus, which was confirmed by CT scanning. Subsequently, the patient underwent an uneventful completion of pancreatectomy. Pathological examination of the surgical specimen confirmed the presence of a 19-mm PDAC with no tumorpositive lymph nodes out of three removed. Molecular analysis of both lesions through Next Generation Sequencing (NGS) showed a class 5 variant in KRAS, NM_033360.2:c.34G>C, p.(Gly12Arg) and class 4 variant in SMAD4, NM_005359.5:c.353C >T, p.(Ala118Val) for the first tumor in the head, and a class 5 variant in KRAS, NM_033360.2:c.35G>T, p.(Gly12Val) together with a class 4 variant in TP53, NM_000546.5:c.517G>T, p.
(Val173Leu) for the second tumor, besides the known p16Leiden variant in $C D K N 2 A$ [8].

Outside the surveillance program, a second patient with a proven CDKN2A-p16-Leiden mutation carrier at the LUMC was found to have a metachronous PDAC. This 57-year-old female underwent a Whipple operation due to an ampullary carcinoma 11 years back. She has never smoked or consumed alcohol. The family history revealed that her mother and maternal grandfather both had ampullary carcinomas previously. A maternal uncle and aunt, and the aunt's children have been diagnosed with melanoma. The patient was presented in March 2017 with liver and pulmonary metastases and a mass at the site of the anastomosis from the Whipple operation. The CA19.9 was $>12,000$. FNA biopsy showed a cancer of pancreaticobiliary origin. Molecular analysis using NGS of the ampullary carcinoma diagnosed 11 years ago revealed class 5 variant in KRAS, NM_033360.2:c.35G>A, p. (Gly12Asp) and the known pl6-Leiden variant in CDKN2A.

In tumor cells collected by fine-needle aspiration (FNA) from the recently diagnosed tumor, NGS also showed the same class 5 variant in KRAS and the p16-Leiden $19 \mathrm{bp}$ deletion. In addition, a class 5 variant was found in TP53, NM_000546.5:c.722C $>$ T, p.(Ser241Phe), a class 3 STK11 missense variant in NM_000455.4/NG_007460.2: c.598$1 \mathrm{G}>\mathrm{A}$, and a deletion of SMAD4, determined by copy number analysis. In view of the identification of the p16Leiden variant, the observed partial differences in the gene variants between the two tumors and the long time-interval since the first cancer, the newly diagnosed tumor should be regarded as a second primary PDAC.

If we include the previously reported cases, we identified two CDKN2A-p16-Leiden patients with multiple PDAC out of the 18 screen-detected PDAC (11\%), one with two synchronous PDAC and one with a metachronous PDAC. Two CDKN2A-p16-Leiden patients detected outside the program were both with metachronous PDAC.

\section{Discussion}

The present study indicates that multiple PDACs can be found in a substantial proportion (up to $11 \%$ ) of $C D K N 2 A$ p16-Leiden mutation carriers. As most patients with hereditary PDAC die within 1 year, the real risk is probably much higher.

The risk of synchronous or metachronous cancers is a well-known phenomenon in inherited forms of cancer $[9,10]$. Owing to the current surveillance program for CDKN2A-p16-Leiden carriers, an increasing number of patients with PDAC are diagnosed at an early stage, resulting in a longer life expectancy [4]. As a consequence, we expect to diagnose more patients with multiple tumors. 
Our findings have immediate clinical implications. Firstly, after detection of a primary PDAC, it is very important to exclude the presence of a second synchronous tumor. As the identification of a second synchronous tumor in patients with sporadic PDAC is very unusual, a second tumor can be easily overlooked, as illustrated by one of the patients presented here. Secondly, in patients with hereditary PDAC undergoing partial resection of a primary tumor, close surveillance (e.g., at six month intervals) of the remaining pancreas is of utmost importance, as three of our patients developed a second PDAC [5, 6].

The most challenging question is whether we should now offer total pancreatectomy to all CDKN2A-p16-Leiden mutation carriers with primary PDAC. In patients with PDAC with poor prognostic indicators, a partial pancreatectomy is probably still the best option because expected survival is usually less than 2 years and a total pancreatectomy would substantially reduce the remaining quality of life. However, patients with a small lesion $(<15 \mathrm{~mm})$ and no evidence of lymph node metastases have a much better prognosis, and total pancreatectomy could be considered. A well-known disadvantage of the total pancreatectomy is the development of diabetes and the associated significant impairment of general quality of life [11]. On the other hand, a disadvantage of a partial resection is that, even with intensive surveillance, the chance of detecting a second tumor at an early stage is limited. For CDKN2A-p16 mutation carriers, a total pancreatectomy may therefore bring relief from the ongoing stress associated with the possibility of a second PDAC. Thus far, we have preferred to offer all patients with screen-detected PDAC a partial pancreatectomy and intensive follow-up after surgery. However, in view of the current findings, offering a total pancreatectomy might be an appropriate option in patients with early PDAC. We therefore suggest that all the pros and cons are discussed with a patient prior to surgery, resulting in a shared decision.

\section{References}

1. Bartsch DK, Gress TM, Langer P. Familial pancreatic cancercurrent knowledge. Nat Rev Gastroenterol Hepatol. 2012;9:445-53.

2. Zhen DB, Rabe KG, Gallinger S, Syngal S, Schwartz AG, Goggins MG, et al. BRCA1, BRCA2, PALB2, and CDKN2A mutations in familial pancreatic cancer: a PACGENE study. Genet Med. 2015;17:569-77.

3. Vasen HF, Gruis NA, Frants RR, van Der Velden PA, Hille ET, Bergman W. Risk of developing pancreatic cancer in families with familial atypical multiple mole melanoma associated with a specific 19 deletion of p16 (p16-Leiden). Int J Cancer. 2000;87:809-11.

4. Vasen H, Ibrahim I, Ponce CG, Slater EP, Matthai E, Carrato A, et al. Benefit of surveillance for pancreatic cancer in high-risk individuals: outcome of long-term prospective follow-up studies from three European expert centers. J Clin Oncol. 2016;34:2010-9.

5. Potjer TP, Bartsch DK, Slater EP, Matthai E, Bonsing BA, Vasen HF. Limited resection of pancreatic cancer in high-risk patients can result in a second primary. Gut. 2015;64:1342-4.

6. Sibinga Mulder BG, Mieog JS, Handgraaf HJ, Farina Sarasqueta A, Vasen HF, Potjer TP, et al. Targeted next-generation sequencing of FNA-derived DNA in pancreatic cancer. J Clin Pathol. 2017;70:174-8.

7. Vasen HF, Wasser M, van Mil A, Tollenaar RA, Konstantinovski M, Gruis NA, et al. Magnetic resonance imaging surveillance detects early-stage pancreatic cancer in carriers of a p16-Leiden mutation. Gastroenterology. 2011;140:850-6.

8. Plon SE, Eccles DM, Easton D, Foulkes WD, Genuardi M, Greenblatt MS, et al. Sequence variant classification and reporting: recommendations for improving the interpretation of cancer susceptibility genetic test results. Hum Mutat. 2008;29:1282-91.

9. Parry S, Win AK, Parry B, Macrae FA, Gurrin LC, Church JM, et al. Metachronous colorectal cancer risk for mismatch repair gene mutation carriers: the advantage of more extensive colon surgery. Gut. 2011;60:950-7.

10. Narod SA. Bilateral breast cancers. Nat Rev Clin Oncol. 2014;11:157-66.

11. Roberts KJ, Blanco G, Webber J, Marudanayagam R, Sutcliffe RP, Muiesan P, et al. How severe is diabetes after total pancreatectomy? A case-matched analysis. HPB. 2014;16:814-21. 